\title{
A Cross Sectional Study on Feeding Practice of New Born Babies in Selected Hospitals
}

\begin{abstract}
Md Monoarul Haque ${ }^{*}$
A K M Mejbah Uddin ${ }^{2}$ Md Rijwan Bhuiyan ${ }^{3}$

A S M Mazharul Islam ${ }^{4}$ Md Shah Billal Masum ${ }^{5}$ Monirul Haqe ${ }^{6}$
\end{abstract}

'Department of Community Nutrition Faculty of Public Health

Bangladesh University of Health Sciences (BUHS) Dhaka, Bangladesh.

${ }^{2}$ Department of Public Health \& Life Sciences University of South Asia

Dhaka, Bangladesh.

${ }^{3}$ Department of Health Promotion and Health Education Bangladesh Institute of Health Sciences (BIHS) Dhaka, Bangladesh.

${ }^{4}$ Health Care Development Project Ibrahim General Hospital

Bangladesh Institute of Health Sciences

Dhaka, Bangladesh.

\section{${ }^{5}$ Senior Lecturer}

Community Medical Institute

Gaibandha, Bangladesh.

\section{${ }^{6}$ Consultant}

Panaroma General Hospital Dhaka, Bangladesh.

\section{*Correspondence to:}

\section{Md Monoarul Haque}

Fellow

Dept of Community Nutrition, Faculty of Public Health, Bangladesh University of Health Sciences (BUHS),

125/1, Darus Salam, Mirpur

Dhaka-1216, Bangladesh.

Mobile: +8801915839550

E-mail: monoarmunna@yahoo.com

\begin{abstract}
Background: Exclusive Breast feeding is implies feeding of breast milk is enough for the baby up to six months of life and no other food is necessary during this time. Objective: The main objective of this study is to study the feeding practice of newborn babies in first week of life. Methods: This study was a cross-sectional study conducted on mothers who have done C-section. About 120 sample were interviewed in Dhaka Medical College Hospital and Pan Pacific Hospital Ltd, Dhaka. Pretested structured questionnaire was used for data collection. Results: The result showed that showed that one-third of the infants $84(70 \%)$ were between the ages of 1 to 2 days and $29(24.2 \%)$ were between the age of 3 to 4 days and the rest $7(5.8 \%)$ were greater than 4 days and about half (53\%) mothers initiate breast feeding within one hour after delivery. Besides study found that more than half of mothers 67(55.8\%) were fed 5-8 time, 40 (33.4\%) mother were fed 8-12 time and 13 $(10.8 \%)$ mothers were fed per day $12-16$ time and about $82 \%$ mothers use water as an extra feed of their child. Conclusion: The study result does not represent the country situation and should not generalize. Further study is needed for more specific information and generalization.
\end{abstract}

Key words: Feeding practice; New born; Caesarean Section.

\section{INTRODUCTION}

Child health in developing countries is a serious concern. A large number of children are still suffering from malnourished. About $43.6 \%$ of under five children in South Asia are underweight ${ }^{1}$. The benefits of breastfeeding for child and mothers in terms of nutrition, immunological protection, anti-infective, biochemical, anti-allergic, anti-cancer and contraceptive effects and emotional satisfaction have been recognized by public health experts and communities. Exclusive Breast feeding is implies feeding of breast milk is enough for the baby up to six months of life and no other food is necessary during this time ${ }^{2}$. The Global Strategy for Infant and Young Child Feeding developed jointly by UNICEF and the WHO and approved by the World Health Assembly in May 2002 provides a strong basis for policy and action on the issue of appropriate feeding practices for infants and young children. In balancing the need for reducing the risk of HIV transmission to infants with the necessity of minimizing the risk of other causes of morbidity and mortality ${ }^{3}$. More frequent breast feedings 8-12 times per day during the first week of life are associated with greater overall milk intake and greater weight gain at 15 days and six weeks of age of the baby ${ }^{4}$. Mothers were also benefited from early suckling because it stimulates breast milk production and facilitates the release of oxytocin, which helps the contraction of the uterus and reduces postpartum blood loss. The first breast milk contains colostrum, which is highly nutritious and has antibodies that protect the newborn from diseases. Early initiation of breastfeeding also encourages bonding between the mother and her newborn. Colostrum is the secretion of breast following child birth for the first 2-3 days build antibody of the body. 
Breast feed infants have enhance immune response and reduce risk for chronic illness such as asthma, diabetes and inflammatory bowel disease. Breast feeding may also have a protective effect against childhood obesity. Further more, breast feed improves maternal health, minimizes post partum bleeding, and reduces the risk of ovarian cancer and breast cancer and. Milk starts to be produced in larger amounts between 2 and 4 days after delivery, making the breasts feel full; the milk is then said to have "come in". On the third day, an infant is normally taking about $300-400 \mathrm{ml}$ per 24 hours, and on the fifth day $500-800 \mathrm{ml}$ from day 7 to 14 , the milk is called transitional, and after 2 weeks it is called mature milk ${ }^{5}$. Prelacteal feeding is widely practiced in Bangladesh. More than $62 \%$ newborns are practiced breastfeeding is almost universal in our our country. Overall, 43 percent of children are breastfed within one hour after birth, and 89 percent are breastfed within one day after delivery. Prelacteal feeds are given to newborns before breastfeeding or before breast milk "comes in," usually on the first day of life. Prelacteals include honey, ghee (clarified butter), and juice, ORS, cows milk and in fant formula.The choice of prelacteals may be specific to a caste or family. The document indicators for assessing published in 1991 provided a set of indicators that could to assess infant feeding within and across countries and evaluate the progress of breastfeeding promotion efforts ${ }^{6}$. In one study it was found that 2,105 mothers in rural Bangladesh, $85 \%$ had fed their child prelacteal food, and only $10 \%$ fed breast milk ${ }^{7}$. Artificially-fed children have an increased risk of long term diseases with an immunological basis. Artificial feeding is also associated with a greater risk of childhood various illness. Breast milk contains all the nutrients that an infant needs in the first 6 months of life, including fat, carbohydrates, proteins, vitamins, minerals and water. It is easily digested and efficiently used. Breast milk also contains bioactive factors that augment the infant's immature immune system, providing protection against infection, and other factors that help digestion and absorption of nutrients. It is produced in small amounts, about $40-50 \mathrm{ml}$ on the first day, but is all that an infant normally needs at this time. Despite declaration of clear benefits of breastfeeding, worldwide breastfeeding rates continue to decline. Exclusive breastfeeding (giving no other food or drink) is even less common ${ }^{2}$. Less than optimal breast-feeding practices have negative impact on child health and family ${ }^{8,9}$. Prelacteal feeding is the practice of giving other liquids to a child during the first three days of life ${ }^{10}$. In addition lack of mother's knowledge about the benefit of the initiation of breast milk (colostrums milk), lack of proper awareness, inadequate nutrition information is the main causes of prelacteal feeding. In our country we have limited information on the feeding practice of newborn babies in first week of life. The present pilot study as an essential part of my dissertation has been undertaken to gather information on feeding practice of newborn.

\section{MATERIALS \& METHOD}

Study design and study place: This was a cross-section study. The study subjects were collected from Dhaka Medical College Hospital and Pan Pacific Hospital Ltd, Dhaka

Subjects: A total of 120 new born babies were collected purposively

Inclusion criteria: New born babies in first week of life and who were delivered by caesarian section

Exclusion criteria: New born baby above 7 days of age and those mother/guardian who refused to participate in interview

Duration of the study: The study was conducted from January 2012 to December 2012

Sampling Technique: Subjects were selected purposively on the basis of availability

Data collection technique: Data were colleted direct interviewing (Face- to- face) through a structure questionnaire by research himself. The questionnaire was prepared in accordance to variable considering the objective of the study. Before data collection, the questionnaire was pre-tested for finalization on the basis of experience obtained from the pretesting and necessary correction and modification was done.

Ethical consideration: All ethical issues, which were related to the research involving human subjects was followed according to the guideline of Bangladesh and WHO research ethical review committee.

Data analysis: After completion of data collection, the collected data were checked and verified daily for accuracy, consistency, and completeness. Data were analyzed according to the objectives of the study objectives of the study. The data were expressed as the frequency, percentage into the form of tables and figures. All statistical analysis were performed with the software SPSS 11.5 for windows (SPSS, Inc IL. USA).

Quality assurance: For quality assurance following points was done:

-Before going for data collection pre test was done

-Recheck of the data before analysis

\section{RESULTS}

Table 1 showed that one-third of the infants 84 (70\%) were between the ages of 1 to 2 days and $29(24.2 \%)$ were between the age of 3 to 4 days and the rest $7(5.8 \%)$ were greater than 4 days. Besides 70 (58.3\%) infants were Boys and 50 (42.7\%) were Girls. Regarding religion $115(96 \%)$ of mothers were Muslim and the rest $3(2 \%)$ Hindu and others were $2(2 \%)$ .One- third mothers $59(49.1 \%)$ were up to class eight, 30 (25\%) mothers were Illiterate, $22(18.3 \%)$ passed S.S.C and rest of them were HSC and above. One-third fathers 47 (39\%) were up to class eight, 29 (24.2\%) husbands were no education and $21(17.5 \%)$ passed SSC, $11(9.2 \%)$ passed HSC and 7 (5.8\%) were graduate. Moreover 115 (95.8\%) mothers were housewife and $3(2.5 \%)$ were service holder. More than half of the family $66(55 \%)$ had monthly income ranging from 500010000 taka and 76 (63\%) family expenditure had ranging 500010000 taka. 
Table 1: Characteristics of the newborn babies and their parents $(n=120)$

\begin{tabular}{lr} 
Characteristics & n(\%) \\
Age & \\
$1-2$ days & $84(70 \%)$ \\
$3-4$ days & $29(24 \%)$ \\
$>4$ days & $7(6 \%)$ \\
Sex & \\
Boys & $70(58 \%)$ \\
Girls & $50(42 \%)$ \\
Religion & \\
Islam & $115(96 \%)$ \\
Hindu & $3(2 \%)$ \\
Others & $2(2 \%)$ \\
Education of Mother & \\
No education & $30(25 \%)$ \\
Up to class eight & $59(49 \%)$ \\
SSC & $22(18 \%)$ \\
HSC & $4(3 \%)$ \\
Graduation & $3(3 \%)$ \\
Others & $2(2 \%)$ \\
Occupation of Mother & \\
Housewife & $115(96 \%)$ \\
Service & $3(2 \%)$ \\
Others & $2(2 \%)$ \\
Monthly Income of the family (BDT) & \\
$\leq 5000$ & \\
$5000-10000$ & $38(32 \%)$ \\
$10000-20000$ & $66(55 \%)$ \\
$20000-50000$ & $6(5 \%)$ \\
$>50000$ & $7(6 \%)$ \\
Monthly expenditure of the family(BDT) & $3(2 \%)$ \\
$\leq 5000$ & \\
$5000-10000$ & $31(26 \%)$ \\
$10000-20000$ & $76(63 \%)$ \\
$20000-50000$ & $9(8 \%)$ \\
\hline & $4(3 \%)$ \\
\hline
\end{tabular}

It was evident from figure 1 that half of the mothers $50(53 \%)$ started breast feeding less than half an hour, 18(18\%) mothers started breast feeding within 2-3 hour after birth, 2(6\%) mothers started breast feeding after 3-6 hour and 32(33\%) mothers started breast feeding after more than 6 hour.

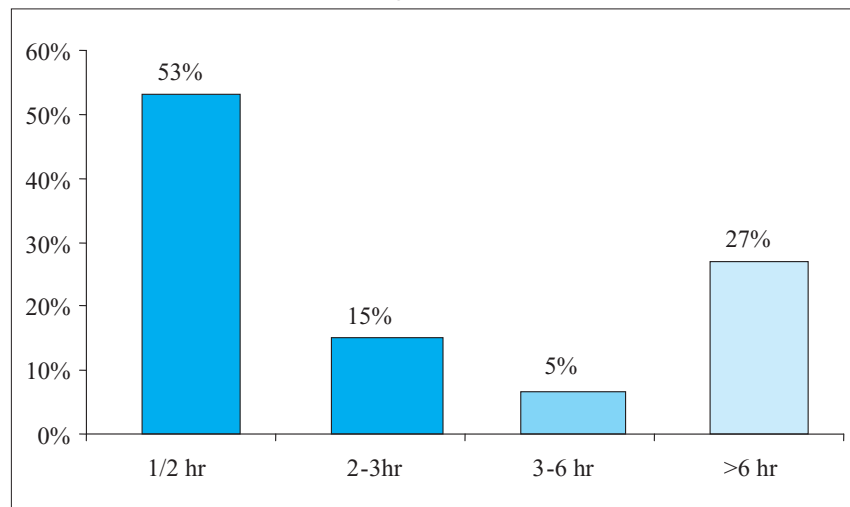

Figure 1: Distribution of the respondents according to the initiation time of breast feeding $(n=120)$
Here showed that more than half of mothers $67(55.8 \%)$ were fed 5-8 time, $40(33.4 \%)$ mother were fed 8-12 time and 13 $(10.8 \%)$ mothers were fed per day 12-16 time.

Table 2: Distribution of the study subjects by the frequency of breast feeding per day $(n=120)$

\begin{tabular}{lcc} 
Times per day & Number & Percentage \\
5-8 time & 67 & 55.8 \\
8- 12 time & 40 & 33.4 \\
12-16 time & 13 & 10.8 \\
\hline
\end{tabular}

Figure 2 indicated various type of food used for prelacteal feeding. Water was more commonly $82(63 \%)$ followed by juice $23(24 \%)$, sugar water $7(3 \%)$, and others $1(7 \%)$.

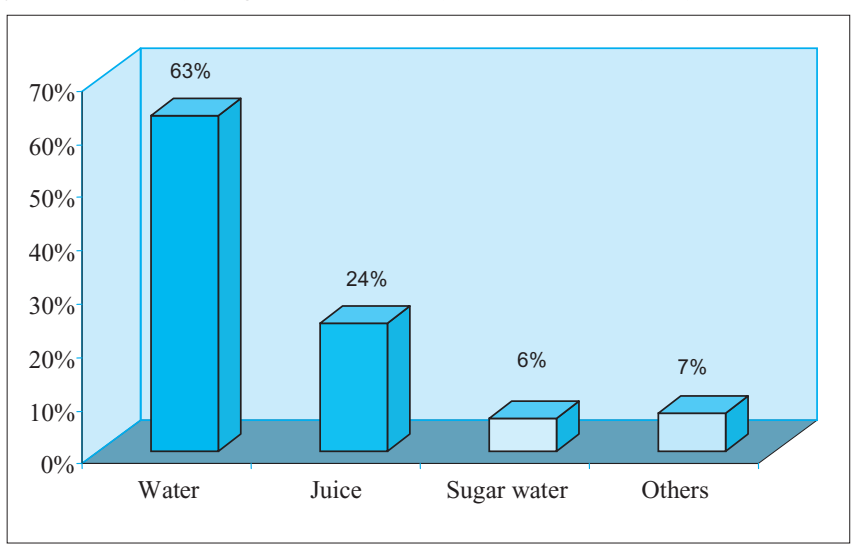

Figure 2: Distribution of the study subjects by prelacteal feeding $(\mathrm{n}=120)$

\section{DISCUSSION}

Study showed that about half of the mothers $(53 \%)$ started breast feeding less than half an hour of delivary, (18\%) mothers started breast feeding within 2-3 hour after birth. According to $\mathrm{WHO}^{5}$ and $\mathrm{UNICEF}^{2}$, early breastfeeding initiation is essential, generally recommended within first hour, it is also beneficial for both the mother and the infant as well. With the initiation of breastfeeding, postpartum hemorrhage is controlled with secreted oxytocin hormone. The first milk, colostrum, contains immunologic and nutritious properties not only to protect the newborn from infections but also to speed up their normal intestinal functions. ${ }^{11}$ Early breastfeeding has been seen to reduce neonatal and postneonatal deaths ${ }^{12}$. Basically cesarean section seems to be a major barrier to early breastfeeding initiation $^{13-16}$. About half of mothers (55.8\%) were fed 5-8 time and about one third (33.4\%) mothers were fed 8-12 time per day. Where water was more commonly (63\%) followed by juice (24\%) used which is not required for new born babies. However, the findings of this study does not represent the actual scenario of the country, although it gives a snap shoot that still now feeding practice after delivery specially after Csection is not in satisfactory rate. Further study is required to find out the actual data and awareness and education program should required to ensure good feeding practice in Bangladesh.

\section{ACKNOWLEDGMENT}

The authors express their sincere thanks to all the patients of this study. No external funding was provided for this study. 


\section{CONCLUSION \& RECOMMENDATION}

The study result does not represent the country situation and should not generalize. Further study is needed for more specific information and generalization.

\section{DISCLOSURE}

All the authors declared no competing interest.

\section{REFERENCES}

1. ACC/SCN Fourth Report on the World Nutrition Situation. Geneva: ACC/SCN in collaboration with IFPRI; 2000

2. Rant JP,editor. State of World Children.3rd edition. UNICEF,NewYork,Oxford University Press;2008.

3. Bendey M.E, Dec. D.L, Jensen J.L. Breast feeding among law income African American Women: Power, Beliefs and Decision Making Journal of Nutrition. 2003;133:305-309.

4. Hussain MA, Khan AK. Studies on the Nutritional status of expectant Mothers \& newborn babies. Bangladesh Medical Research council Bulletin.1976; 2 (2): 120-126.

5. World Health Organization. Infant and Young child feeding; 2009.

6. World Health Organization. Indicators for assessing infant and young child feeding practices, November 2008.

7. World Health Organization. Child growth standards adopted survey ; BDHS 2007.

8. Rasheed S, Biag LA. et al. Decline in Breast Feeding, Who is to be blamed? JPMA. 2002;50:8.

9. Hussain MA, Khan AK. Studies on the Nutritional status of expectant Mothers \& newborn babies. Bangladesh Medical Research council Bulletin, 1976; 2 (2): 120-126.

10. Hoque N, Feeding Pattern of Newborn Baby Delivered by Caesarean Section During Hospital Stay [Dissertation]. MPH, NIPSOM; June 2004.

11. Gartner LM, Morton J, Lawrence RA, et al. American Academy of Pediatrics Section on Breastfeeding. Breastfeeding and the use of human milk. Pediatrics. 2005; 115: 496-506.

12. Edmond KM, Zandoh C, Qigley MA, Amenga-Etego S, Owusu-Agyei S, Kirkwood BR. Delayed breastfeeding initiation increases risk of neonatal mortality. Pediatrics. 2006; 117: e380-e386.

13. Awi DD, Alikor EA. Barriers to timely initiation of breastfeeding among mothers of healthy full-term babies who deliver at the University of Port Harcourt Teaching Hospital. Niger J Clin Pract. 2006; 9: 57-64.

14. Nakao Y, Moji K, Honda S, Oishi K. Initiation of breastfeeding within 120 minutes after birth is associated with breastfeeding at four months among Japanese women: a self-administered questionnaire survey. Int Breastfeed J. 2008; 3: 1-7.

15. Kovach AC. A 5-year follow-up study of breastfeeding policies in the Philadelphia area: a comparison with the ten steps. J Hum Lact. 2002; 18: 144-154.

16. Rowe-Murray HJ, Fisher JR. Baby friendly hospital practices: cesarean section is a persistent barrier to early initiation of breastfeeding. Birth. 2002; 29: 124-131 